\title{
Intrinsic localized modes in coupled DNLS equations from the anti-continuum limit
}

\author{
K. Li ${ }^{\text {a }}$ P.G. Kevrekidis ${ }^{a}$ H. Susanto ${ }^{b}$ V. Rothos ${ }^{c}$ \\ ${ }^{a}$ Department of mathematics and statistics, university of Massachusetts, Amherst, \\ Massachusetts 01003-4515, USA \\ ${ }^{\mathrm{b}}$ School of mathematical sciences, university of Nottingham, University Park, \\ Nottingham, NG7 2RD, United Kingdom \\ ${ }^{\mathrm{c}}$ Department of mathematics, physics and computational sciences, faculty of \\ engineering, Aristotle university of Thessaloniki, Thessaloniki 54124, Greece
}

\begin{abstract}
In the present work, we generalize earlier considerations for intrinsic localized modes consisting of a few excited sites, as developed in the one-component discrete nonlinear Schrödinger equation model, to the case of two-component systems. We consider all the different combinations of "up" (zero phase) and "down" ( $\pi$ phase) site excitations and are able to compute not only the corresponding existence curves, but also the eigenvalue dependence of the small eigenvalues potentially responsible for instabilities, as a function of the nonlinear parameters of the model representing the self/cross phase modulation in optics and the scattering length ratios in the case of matter waves in optical lattices. We corroborate these analytical predictions by means of direct numerical computations. We infer that all the modes which bear two adjacent nodes with the same phase are unstable in the two component case and the only solutions that may be linear stable are ones where each set of adjacent nodes, in each component is out of phase.
\end{abstract}

\section{Introduction}

The theme of nonlinear dynamical lattices and especially of the prototypical equation of the Discrete Nonlinear Schrödinger (DNLS) type [17] has been a widely studied one over the last decade, perhaps especially so due to its applications in both the field of nonlinear optics, as well as in that of atomic physics.

The realization of this type of models in the context of matter waves arose due to the examination of the dynamics of Bose-Einstein condensates (BECs) 
in periodic, so-called optical lattice, potentials. In that realm, the reduction of the prototypical mean-field model of the Gross-Pitaevskii equation with a periodic potential, in the superfluid regime, via a tight binding approximation, naturally yields the DNLS as the canonical lattice model for the BEC "droplets" [3,26,18].

On the other hand, the nonlinear optics realization in fabricated AlGaAs waveguide arrays [9] has been one that preceded the atomic physics realization and yielded numerous insights about the interplay of discrete lattice dynamics with the effects of nonlinearity, including but not limited to PeierlsNabarro barriers and discrete solitons, modulational instabilities, gap solitons, diffraction and diffraction management; see e.g. $[2,11,10,5,20]$ for a number of relevant reviews. Another area of optical applications which has seen considerable development over the past decade is that of optically induced lattices in photorefractive crystals such as Strontium-Barium-Niobate [8,14,13]. Although the latter setting is not directly modeled by the DNLS (chiefly due to the photorefractive, saturable nature of the nonlinearity), it has enabled the experimental observation of numerous solitary/localized patterns that were predicted in the context of the DNLS; see e.g. the reviews $[20,12]$.

A topic that has been of particular interest in both the atomic and the optical applications has been that of multi-component dynamics. Among the many recent experimental results related to the interaction of two different frequencies or polarizations in optics or of two hyperfine atomic states or two different atoms in BEC, we highlight only a few. The first experimental observations of discrete vector solitons in waveguide arrays were reported in [22], while the emergence of multipole patterns in two-component settings within photorefractive crystals was presented in [4], and more recently more complex patterns including even dark-bright lattice solitons were reported experimentally [7]. In the BEC case, experiments with mixtures of different spin states of ${ }^{87} \mathrm{Rb}[28,23]$ or ${ }^{23} \mathrm{Na}[30]$ and even ones of different atomic species such as ${ }^{41} \mathrm{~K}-$ ${ }^{87} \mathrm{Rb}[24]$ and ${ }^{7} \mathrm{Li}-{ }^{133} \mathrm{Cs}[27]$ have been realized and although localized modes in optical lattices have not been probed in the form considered herein, certainly such experiments appear to be within the current experimental reach [25].

Our scope in the present communication is to explore systematically the existence and stability properties of two-component localized modes, motivated by the above applications. The most fundamental excitations (single site) in the coupled DNLS equations model were explored a considerable while ago not only in one- but also in higher dimensions; see for a relevant discussion [16] and references therein. In fact, in higher dimensions also vortex states were considered [19]. However, in the one-dimensional realm, we are not aware of a multi-component generalization of the theory of [29] (for the existence theory, see also [1]) which enables the systematic quantification of the existence and stability properties of localized modes consisting of an arbitrary number of 
sites. Here, we present the theory of how to address the problem of existence and stability in the two-component setting, which paves the way also towards the generalization to a higher number of components. Aside from providing the general theory, we consider numerous special case examples involving excitation of two- and also of three-sites in both components with either zero phase (an "up" site) or with $\pi$ phase (a "down" site). For all of these configurations we examine their stability properties as a function, e.g., of their nonlinear coefficients (dubbed $g_{i j}$ ), which stand for the self- and cross-phase modulation parameters in optics and for the scattering lengths (or more accurately their ratios) in the realm of BEC. Interestingly, we find that independently of the nature of the second component and of the inter- and intra-component coupling strengths, should two adjacent sites in any one component be of the same phase, the configuration is found to be unstable. The only potentially stable configurations consist of nodes with alternating adjacent phases, similarly to what is known for the one-component problem [29].

Our presentation is structured as follows. Section II presents the general existence and stability theory. Section III examines special case examples of configurations. Finally, section IV summarizes our findings and presents our conclusions, as well as topics for potential further studies.

\section{General theory}

Our fundamental model will be that of the two coupled DNLS equations (see e.g. $[16,29,6])$ of the form:

$$
\left\{\begin{array}{l}
i \dot{U}_{n}+\left(g_{11}\left|U_{n}\right|^{2}+g_{12}\left|V_{n}\right|^{2}\right) U_{n}+\epsilon \Delta U_{n}=0 \\
i \dot{V_{n}}+\left(g_{12}\left|U_{n}\right|^{2}+g_{22}\left|V_{n}\right|^{2}\right) V_{n}+\epsilon \Delta V_{n}=0
\end{array}\right.
$$

The localized modes (often referred to also as "discrete solitons") are given by the time-periodic solutions of the DNLS equation

$$
\left\{\begin{array}{l}
U_{n}(t)=\phi_{n} e^{i(\mu-2 \epsilon) t+i \xi_{0}} \\
V_{n}(t)=\psi_{n} e^{i(\mu-2 \epsilon) t+i \zeta_{0}}
\end{array}\right.
$$

where $\mu \in \mathbb{R}, \phi_{n}, \psi_{n} \in \mathbb{C}, n \in \mathbb{Z}, \xi_{0}, \zeta_{0} \in \mathbb{R}$ is a parameter and $\left(\mu, \phi_{n}, \psi_{n}\right)$ solve the nonlinear difference equations on $n \in \mathbb{Z}$ : 


$$
\left\{\begin{array}{l}
\left(\mu-g_{11}\left|\phi_{n}\right|^{2}-g_{12}\left|\psi_{n}\right|^{2}\right) \phi_{n}=\epsilon\left(\phi_{n+1}+\phi_{n-1}\right) \\
\left(\mu-g_{12}\left|\phi_{n}\right|^{2}-g_{22}\left|\psi_{n}\right|^{2}\right) \psi_{n}=\epsilon\left(\psi_{n+1}+\psi_{n-1}\right) .
\end{array}\right.
$$

Notice that here we start by examining the simpler case where the soliton parameter $\mu$ (representing the chemical potential in the atomic physics case and the propagation constant in the optical case) is identical in the two components. Nevertheless, this is not necessarily the case given the nonlinear nature of the coupling and the presence of an invariance under phase transformations in each of the involved components. We will comment on the case with distinct values of $\mu$ at the final section of this presentation.

Our principal existence result can be formulated as follows (the proof follows a line directly analogous to that of [29] for the two-component case, imposing the relevant solvability conditions that in turn lock the adjacent site phase differences to the $0, \pi$ relative phases discussed below)

Assume that $\mu=1$ and $\left(g_{12}-g_{22}\right)\left(g_{12}^{2}-g_{11} g_{22}\right)>0$, as well as $\left(g_{12}-g_{11}\right)\left(g_{12}^{2}-\right.$ $\left.g_{11} g_{22}\right)>0$. There exists $\epsilon_{0}>0$ such that the solution $\phi_{n}$ and $\psi_{n}, n \in \mathbb{Z}$, are represented by the convergent power series for $0 \leq \epsilon<\epsilon_{0}$ :

$$
\phi_{n}=\phi_{n}^{(0)}+\sum_{k=1}^{\infty} \epsilon^{k} \phi_{n}^{(k)}
$$

and

$$
\psi_{n}=\psi_{n}^{(0)}+\sum_{k=1}^{\infty} \epsilon^{k} \psi_{n}^{(k)}
$$

where

$$
\left(\begin{array}{c}
\phi_{n}^{(0)} \\
\psi_{n}^{(0)}
\end{array}\right)=\left(\begin{array}{l}
\sqrt{\frac{g_{12}-g_{22}}{g_{12}^{2}-g_{11} g_{22}}} e^{i \xi_{n}} \\
\sqrt{\frac{g_{12}-g_{11}}{g_{12}^{2}-g_{11} g_{22}}} e^{i \zeta_{n}}
\end{array}\right)
$$

or

$$
\left(\begin{array}{c}
\phi_{n}^{(0)} \\
\psi_{n}^{(0)}
\end{array}\right)=\left(\begin{array}{c}
\frac{1}{\sqrt{g_{11}}} e^{i \xi_{n}} \\
0
\end{array}\right)
$$

or

$$
\left(\begin{array}{c}
\phi_{n}^{(0)} \\
\psi_{n}^{(0)}
\end{array}\right)=\left(\begin{array}{c}
0 \\
\frac{1}{\sqrt{g_{22}}} e^{i \zeta_{n}}
\end{array}\right)
$$


with $\xi_{n}, \zeta_{n} \in\{0, \pi\}$, is the solution for

$$
\left\{\begin{array}{l}
\left(1-g_{11}\left|\phi_{n}\right|^{2}-g_{12}\left|\psi_{n}\right|^{2}\right) \phi_{n}=0 \\
\left(1-g_{12}\left|\phi_{n}\right|^{2}-g_{22}\left|\psi_{n}\right|^{2}\right) \psi_{n}=0
\end{array}\right.
$$

for $n \in S=S_{\phi}=S_{\psi}=\{1,2, \cdots, N\}$ which is a finite set of (excited) nodes of the lattice, and $\phi_{n}^{(0)}=\psi_{n}^{(0)}=0$ otherwise.

The above analytical expressions fully describe the anti-continuum limit of uncoupled sites (i.e., $\epsilon=0$ ). In particular, they correspond to the cases of both fields being excited at each lattice node, or of those of one field being excited only thereon. The latter case was considered previously in [6], hence in the present work, we will focus on genuine two-component excitations. Notice also that in the above considerations (and in what follows), we have set, without loss of generality, $\mu=1$. Lastly, we should point out that for the two-excited-component solutions to be meaningful, it should be the case that $\left(g_{12}-g_{22}\right)\left(g_{12}^{2}-g_{11} g_{22}\right)>0$ and similarly that $\left(g_{12}-g_{11}\right)\left(g_{12}^{2}-g_{11} g_{22}\right)>0$. This condition stems from the necessity that the solutions of Eq. (9) for $\left|\phi_{n}\right|^{2}$ and $\left|\psi_{n}\right|^{2}$ be positive. It should be added, however, that these inequalities should not necessarily be thought of as binding either since if the opposite inequality signs hold, then one can simply consider the case where $\mu$ has opposite sign.

The spectral stability of discrete solitons is studied with the standard linearization

$$
\left\{\begin{array}{l}
u_{n}(t)=e^{i(1-2 \epsilon) t+i \xi_{0}}\left(\phi_{n}+a_{n} e^{\lambda t}+\bar{b}_{n} e^{\bar{\lambda} t}\right) \\
v_{n}(t)=e^{i(1-2 \epsilon) t+i \zeta_{0}}\left(\psi_{n}+c_{n} e^{\lambda t}+\bar{d}_{n} e^{\bar{\lambda} t}\right)
\end{array}\right.
$$

where $\lambda, a_{n}, b_{n}, c_{n}, d_{n}$ solve the linear eigenvalue problem on $n \in \mathbb{Z}$ :

$$
\begin{aligned}
& \alpha_{n} a_{n}-g_{11} \phi_{n}^{2} b_{n}-g_{12} \phi_{n} \psi_{n}\left(c_{n}+d_{n}\right)-\epsilon\left(a_{n+1}+a_{n-1}\right)=i \lambda a_{n} \\
& \alpha_{n} b_{n}-g_{11} \phi_{n}^{2} a_{n}-g_{12} \phi_{n} \psi_{n}\left(c_{n}+d_{n}\right)-\epsilon\left(b_{n+1}+b_{n-1}\right)=-i \lambda b_{n} \\
& \beta_{n} c_{n}-g_{22} \psi_{n}^{2} c_{n}-g_{12} \phi_{n} \psi_{n}\left(a_{n}+b_{n}\right)-\epsilon\left(c_{n+1}+c_{n-1}\right)=i \lambda c_{n} \\
& \beta_{n} d_{n}-g_{22} \psi_{n}^{2} d_{n}-g_{12} \phi_{n} \psi_{n}\left(a_{n}+b_{n}\right)-\epsilon\left(d_{n+1}+d_{n-1}\right)=-i \lambda d_{n},
\end{aligned}
$$

where $\alpha_{n}=1-2 g_{11} \phi_{n}^{2}-g_{12} \psi_{n}^{2}, \beta_{n}=1-2 g_{22} \psi_{n}^{2}-g_{12} \phi_{n}^{2}$.

Let $\Omega=l^{2}(\mathbb{Z}, \mathbb{C})$ be the Hilbert space of square-summable bi-infinite complex- 
valued sequences $\left\{u_{n}\right\}_{n \in \mathbb{Z}}$, equipped with the inner product and norm

$$
(\mathbf{u}, \mathbf{v})_{\Omega}=\sum_{n \in \mathbb{Z}} \bar{u}_{n} v_{n}, \quad\|\mathbf{u}\|_{\Omega}^{2}=\sum_{n \in \mathbb{Z}}\left|u_{n}\right|^{2}<\infty .
$$

We use bold notation $\mathbf{u}$ for an infinite-dimensional vector in $\Omega$ that consists of components $u_{n}$ for all $n \in \mathbb{Z}$.

The stability problem (11) is transformed through the substitution

$$
a_{n}=r_{n}+i s_{n}, b_{n}=r_{n}-i s_{n}, c_{n}=p_{n}+i q_{n}, d_{n}=p_{n}-i q_{n}
$$

to the form

$$
\begin{aligned}
& \left(1-3 g_{11} \phi_{n}^{2}-g_{12} \psi_{n}^{2}\right) r_{n}-2 g_{12} \phi_{n} \psi_{n} p_{n}-\epsilon\left(r_{n+1}+r_{n-1}\right)=-\lambda s_{n} \\
& \left(1-g_{11} \phi_{n}^{2}-g_{12} \psi_{n}^{2}\right) s_{n}-\epsilon\left(s_{n+1}+s_{n-1}\right)=\lambda r_{n} \\
& \left(1-3 g_{22} \psi_{n}^{2}-g_{12} \phi_{n}^{2}\right) p_{n}-2 g_{12} \phi_{n} \psi_{n} r_{n}-\epsilon\left(p_{n+1}+p_{n-1}\right)=-\lambda q_{n} \\
& \left(1-g_{22} \psi_{n}^{2}-g_{12} \phi_{n}^{2}\right) q_{n}-\epsilon\left(q_{n+1}+q_{n-1}\right)=\lambda p_{n} .
\end{aligned}
$$

The matrix-vector form of the problem is then

$$
\begin{aligned}
\mathcal{L}_{+} \mathbf{u} & =-\lambda \mathbf{v} \\
\mathcal{L}_{-} \mathbf{v} & =\lambda \mathbf{u},
\end{aligned}
$$

where

$$
\mathbf{u}=\left(\begin{array}{c}
r_{n} \\
p_{n}
\end{array}\right), \mathbf{v}=\left(\begin{array}{c}
s_{n} \\
q_{n}
\end{array}\right),
$$

and $\mathcal{L}_{ \pm}$are infinite-dimensional symmetric matrices which have tridiagonal blocks as follows:

$$
\begin{gathered}
\left(\mathcal{L}_{+}\right)_{n, n}=\left(\begin{array}{cc}
1-3 g_{11} \phi_{n}^{2}-g_{12} \psi_{n}^{2} & -2 g_{12} \phi_{n} \psi_{n} \\
-2 g_{12} \phi_{n} \psi_{n} & 1-3 g_{22} \psi_{n}^{2}-g_{12} \phi_{n}^{2}
\end{array}\right) \\
\left(\mathcal{L}_{-}\right)_{n, n}=\left(\begin{array}{cc}
1-g_{11} \phi_{n}^{2}-g_{12} \psi_{n}^{2} & 0 \\
0 & 1-g_{22} \psi_{n}^{2}-g_{12} \phi_{n}^{2}
\end{array}\right) \\
\left(\mathcal{L}_{ \pm}\right)_{n, n+1}=\left(\mathcal{L}_{ \pm}\right)_{n+1, n}=\left(\begin{array}{cc}
-\epsilon & 0 \\
0 & -\epsilon
\end{array}\right) .
\end{gathered}
$$


Equivalently, the stability problem (15) is rewritten in the Hamiltonian form

$$
\mathcal{J H} \boldsymbol{\eta}=\lambda \boldsymbol{\eta}
$$

where $\boldsymbol{\eta}$ is the infinite-dimensional eigenvector, which consists of 4 -blocks of $\left(r_{n}, p_{n}, s_{n}, q_{n}\right)^{T}, \mathcal{J}$ is the infinite-dimensional skew-symmetric matrix, which consists of 4 -by-4 blocks of

$$
\mathcal{J}_{n, m}=\left(\begin{array}{cccc}
0 & 0 & 1 & 0 \\
0 & 0 & 0 & 1 \\
-1 & 0 & 0 & 1 \\
0 & -1 & 0 & 0
\end{array}\right) \delta_{n, m}
$$

and $\mathcal{H}$ is the infinite-dimensional symmetric matrix, which consists of 2 -by-2 blocks of

$$
\mathcal{H}_{n, m}=\left(\begin{array}{cc}
\left(\mathcal{L}_{+}\right)_{n, m} & 0 \\
0 & \left(\mathcal{L}_{-}\right)_{n, m}
\end{array}\right)
$$

The representation (18) follows from the Hamiltonian structure of the DNLS equation (1), where $\mathcal{J}$ is the symplectic operator and $\mathcal{H}$ is the linearized Hamiltonian. Using our above existence results, the matrix $\mathcal{H}$ can be expanded (again for $\epsilon$ small) into the power series

$$
\mathcal{H}=\mathcal{H}^{(0)}+\sum_{k=1}^{\infty} \epsilon^{k} \mathcal{H}^{(k)}
$$

where

$$
\begin{gathered}
\mathcal{H}_{n, n}^{(0)}=\left(\begin{array}{cccc}
\left.-2 g_{11} \phi_{n}^{(0)}\right)^{2} & -2 g_{12} \phi_{n}^{(0)} \psi_{n}^{(0)} & 0 & 0 \\
-2 g_{12} \phi_{n}^{(0)} \psi_{n}^{(0)} & \left.-2 g_{22} \psi_{n}^{(0)}\right)^{2} & 0 & 0 \\
0 & 0 & 0 & 0 \\
0 & 0 & 0 & 0
\end{array}\right), \quad n \in S, \\
\mathcal{H}_{n, n}^{(0)}=\left(\begin{array}{llll}
1 & 0 & 0 & 0 \\
0 & 1 & 0 & 0 \\
0 & 0 & 1 & 0 \\
0 & 0 & 0 & 1
\end{array}\right), \\
n \in \mathbb{Z} \backslash S .
\end{gathered}
$$

The solution of the difference equations is defined by the power series (4) and (5), and the pair $\phi_{n}^{(1)}, \psi_{n}^{(1)}$ solves the inhomogeneous problem 


$$
\left\{\begin{array}{l}
\left(1-3 g_{11} \phi_{n}^{(0)^{2}}-g_{12} \psi_{n}^{(0)^{2}}\right) \phi_{n}^{(1)}-2 g_{12} \phi_{n}^{(0)} \psi_{n}^{(0)} \psi_{n}^{(1)}=\phi_{n+1}^{(0)}+\phi_{n-1}^{(0)} \\
\left(1-3 g_{22} \psi_{n}^{(0)^{2}}-g_{12} \phi_{n}^{(0)^{2}}\right) \psi_{n}^{(1)}-2 g_{12} \phi_{n}^{(0)} \psi_{n}^{(0)} \phi_{n}^{(1)}=\psi_{n+1}^{(0)}+\psi_{n-1}^{(0)}
\end{array}\right.
$$

The symmetric matrix $\mathcal{H}$ is defined by $(20)$, where $\mathcal{H}^{(0)}$ is given by $(22)$ and $\mathcal{H}^{(1)}$ consists of blocks:

$$
\mathcal{H}_{n, n}^{(1)}=\left(\begin{array}{cc}
\left(\mathcal{L}_{+}^{(1)}\right)_{n, n} & 0 \\
0 & \left(\mathcal{L}_{-}^{(1)}\right)_{n, n}
\end{array}\right)
$$

where

$$
\begin{gathered}
\left(\mathcal{L}_{+}^{(1)}\right)_{n, n}=\left(\begin{array}{cc}
-6 g_{11} \phi_{n}^{(0)} \phi_{n}^{(1)}-2 g_{12} \psi_{n}^{(0)} \psi_{n}^{(1)} & -2 g_{12}\left(\phi_{n}^{(0)} \psi_{n}^{(1)}+\psi_{n}^{(0)} \phi_{n}^{(1)}\right) \\
-2 g_{12}\left(\phi_{n}^{(0)} \psi_{n}^{(1)}+\psi_{n}^{(0)} \phi_{n}^{(1)}\right) & -2 g_{12} \phi_{n}^{(0)} \phi_{n}^{(1)}-6 g_{22} \psi_{n}^{(0)} \psi_{n}^{(1)}
\end{array}\right), \\
\left(\mathcal{L}_{-}^{(1)}\right)_{n, n}=\left(\begin{array}{cc}
-2 g_{11} \phi_{n}^{(0)} \phi_{n}^{(1)}-2 g_{12} \psi_{n}^{(0)} \psi_{n}^{(1)} & 0 \\
0 & -2 g_{22} \psi_{n}^{(0)} \psi_{n}^{(1)}-2 g_{12} \phi_{n}^{(0)} \phi_{n}^{(1)}
\end{array}\right), \\
\mathcal{H}_{n, n+1}^{(1)}=\mathcal{H}_{n+1, n}^{(1)}=\left(\begin{array}{cccc}
-1 & 0 & 0 & 0 \\
0 & -1 & 0 & 0 \\
0 & 0 & -1 & 0 \\
0 & 0 & 0 & -1
\end{array}\right)
\end{gathered}
$$

while all other blocks of $\mathcal{H}_{n, m}^{(1)}$ are zero.

Now, the non-self-adjoint eigenvalue problem (15) can be transformed as:

$$
\mathcal{M} \mathbf{u}=\mathcal{L}_{+} \mathcal{L}_{-} \mathbf{u}=-\lambda^{2} \mathbf{u} .
$$

According to (22),

$$
\begin{gathered}
\left(\mathcal{L}_{+}^{(0)}\right)_{n, n}=\left(\begin{array}{cc}
-2 g_{11} \phi_{n}^{(0)} & -2 g_{12} \phi_{n}^{(0)} \psi_{n}^{(0)} \\
-2 g_{12} \phi_{n}^{(0)} \psi_{n}^{(0)} & -2 g_{22} \psi_{n}^{(0)}{ }^{2}
\end{array}\right), \\
\left(\mathcal{L}_{-}^{(0)}\right)_{n, n}=\left(\begin{array}{ll}
0 & 0 \\
0 & 0
\end{array}\right) .
\end{gathered}
$$

Therefore,

$$
\mathcal{M}^{(1)}=\mathcal{L}_{+}^{(0)} \mathcal{L}_{-}^{(1)},
$$


where $\mathcal{L}_{+}^{(0)}$ is given by $(29)$ and $\mathcal{L}_{-}^{(1)}$ is given by $(26)$, while the expansion $\mathcal{M}=\epsilon \mathcal{M}^{(1)}+O\left(\epsilon^{2}\right)$ has been used. This expansion is a natural generalization of the one-component results of [29]. As a result, if we identify the eigenvalues $\gamma$ of $\mathcal{M}^{(1)}$, we can then trace the eigenvalues of the full Hamiltonian problem according to $\lambda^{2}=-\epsilon \gamma$.
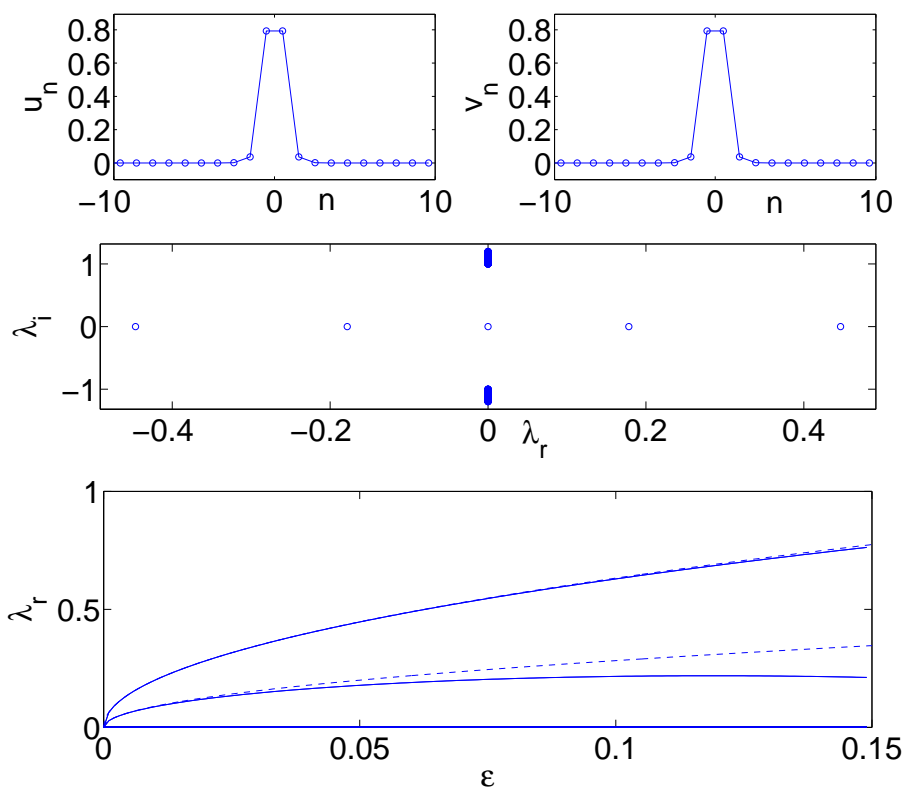

Fig. 1. The top panel shows the spatial profile of the mode,++++ in both components and the corresponding spectral plane of the linear stability problem is shown in the middle panel for $\epsilon=0.05$. The bottom subplot shows the continuation of the branch from $\epsilon=0$ to $\epsilon=0.15$ and the real positive eigenvalue numerically obtained (solid line) and theoretically predicted (dashed line). The theory yields $\lambda^{2}=4 \epsilon, \frac{4}{5} \epsilon$.

We now turn to a number of special case examples, which allow us to consider the stability of the different two-site and three-site configurations.

\section{Case examples}

We now focus on the set $S: N=2$ and $N=3$. Since the phases of the two components assume the values $\xi_{n}, \zeta_{n}=\{0, \pi\}$ for $n \in S=\{1,2, \cdots, N\}$, we use the symbolism "+" for 0 , "-" for $\pi$ and "0" for the case where no excitation is present on a particular site.

When $N=2$, the eigenvalue problem can be explicitly expressed in terms of $g_{11}, g_{12}$ and $g_{22}$ in the eigenvalues of Eq. (31). More specifically, we obtain the following results.

-,++++ : The nonzero eigenvalues of $\mathcal{M}^{(1)}$ are given explicitly as 

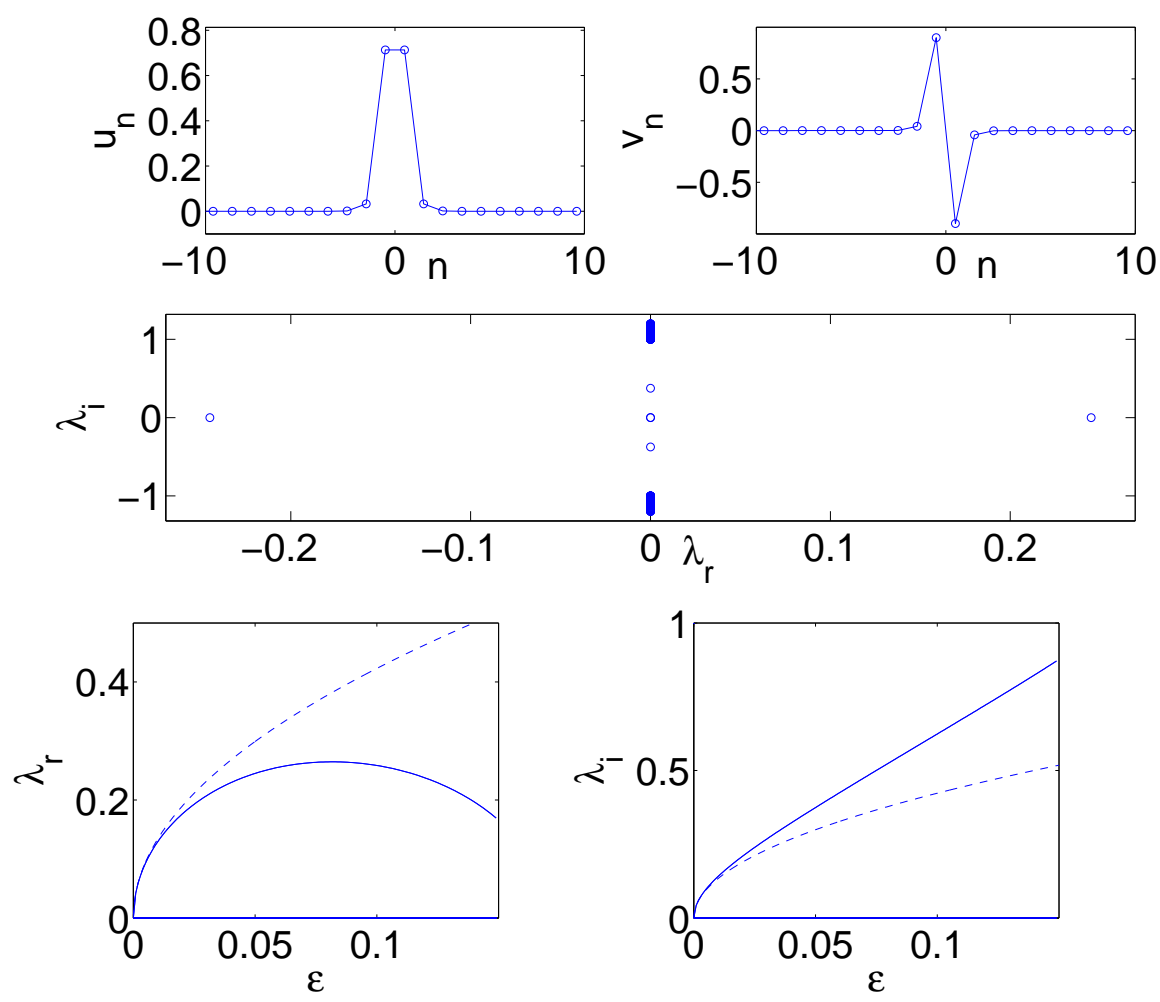

Fig. 2. The top panel shows the mode,+++- and the middle panel its spectral plane for $\epsilon=0.05$. The bottom subplot shows the real and imaginary parts of the eigenvalues (again numerics in solid vs. theory in dashed). The theoretical prediction gives $\lambda^{2}= \pm \frac{4}{\sqrt{5}} \epsilon$.

$$
\begin{aligned}
\gamma_{1} & =\frac{4\left(g_{11}-g_{12}\right)\left(g_{12}-g_{22}\right)}{-g_{12}^{2}+g_{11} g_{22}} \\
\gamma_{2} & =-4 .
\end{aligned}
$$

Therefore, the,++++ mode has two real unstable eigenvalues

$$
\begin{aligned}
& \lambda_{1}=\sqrt{\frac{4\left(g_{11}-g_{12}\right)\left(g_{12}-g_{22}\right)}{g_{12}^{2}-g_{11} g_{22}} \epsilon} \\
& \lambda_{2}=2 \sqrt{\epsilon}
\end{aligned}
$$

in the stability problem (18) for small $\epsilon>0$. Fig 1 shows the result under parameters $g_{11}=g_{22}=1$ and $g_{12}=2 / 3$, where the agreement with theory is very good for $\epsilon<0.05$. We have generally observed this kind of agreement with the analytical results presented herein for an interval of $\epsilon$ values in the vicinity of the anti-continuum limit. As a final observation, associated with this case, we should point out that although the relevant eigenvalue expression has non-trivial zero crossings, the constraints for the existence of a real solution mandate that the relevant eigenvalue always stay real and 

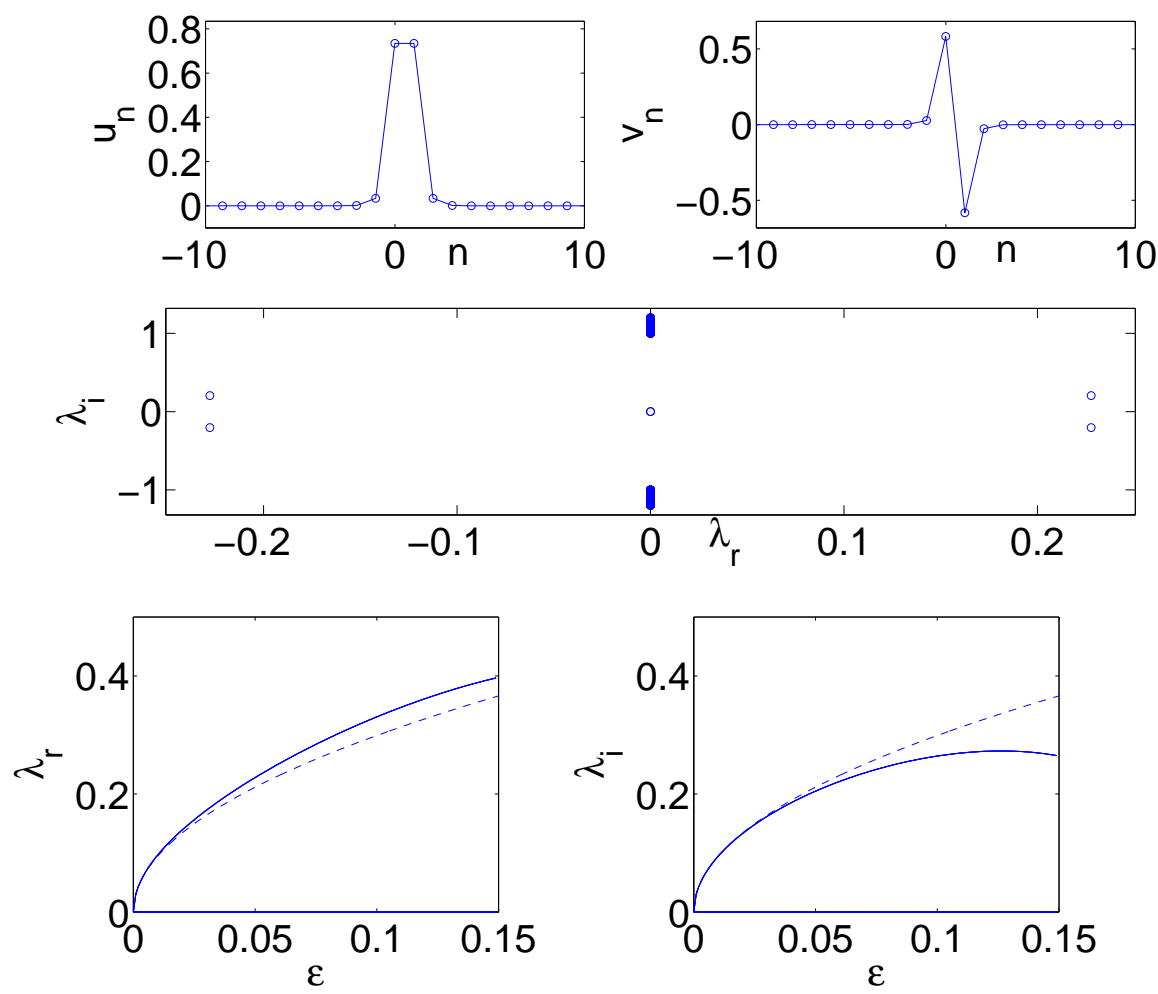

Fig. 3. The top panels show the mode,+++- but with $g_{11}=g_{22}=1, g_{12}=1.5$ and the middle panel its spectral plane for $\epsilon=0.05$. The bottom subplot shows the real and imaginary part of the quartet, for which the theoretical prediction is $\lambda^{2}= \pm \frac{4 i}{\sqrt{5}} \epsilon$.

hence the configuration be unstable ${ }^{1}$.

-,+++- In this case, we can obtain that

$$
\begin{aligned}
\lambda_{1,2}^{2} & =\epsilon \frac{2\left(g_{11} g_{12}-g_{12} g_{22}\right)}{g_{12}^{2}-g_{11} g_{22}} \\
& \pm \epsilon \frac{\sqrt{4\left(g_{11}-g_{12}\right)\left(g_{12}-g_{22}\right)\left(g_{12}^{2}-g_{11} g_{22}\right)+\left(g_{11} g_{12}-g_{12} g_{22}\right)^{2}}}{g_{12}^{2}-g_{11} g_{22}} .
\end{aligned}
$$

In this setting, an interesting possibility arises, which deviates from what is known about the one-component cases. In particular, the nature of the instability of such configurations depends on the choice of $g_{11}, g_{12}$ and $g_{22}$. When $g_{11}=g_{22}$ and $g_{12}>g_{11}$, there is a quartet of complex conjugate eigenvalues which gives rise to an oscillatory instability, as soon as $\epsilon$ becomes nonzero, shown in Fig 3. On the other hand, when $g_{11}=g_{22}$ and $g_{12}<g_{11}$, a pair of real eigenvalues and a pair of purely imaginary eigenvalues are

$\overline{1}$ Recall that in the Hamiltonian system under consideration here, when $\lambda$ is an eigenvalue, so are $-\lambda, \lambda^{\star}$ and $-\lambda^{\star}$, hence the existence of a real eigenvalue pair is always associated with an exponential instability. 

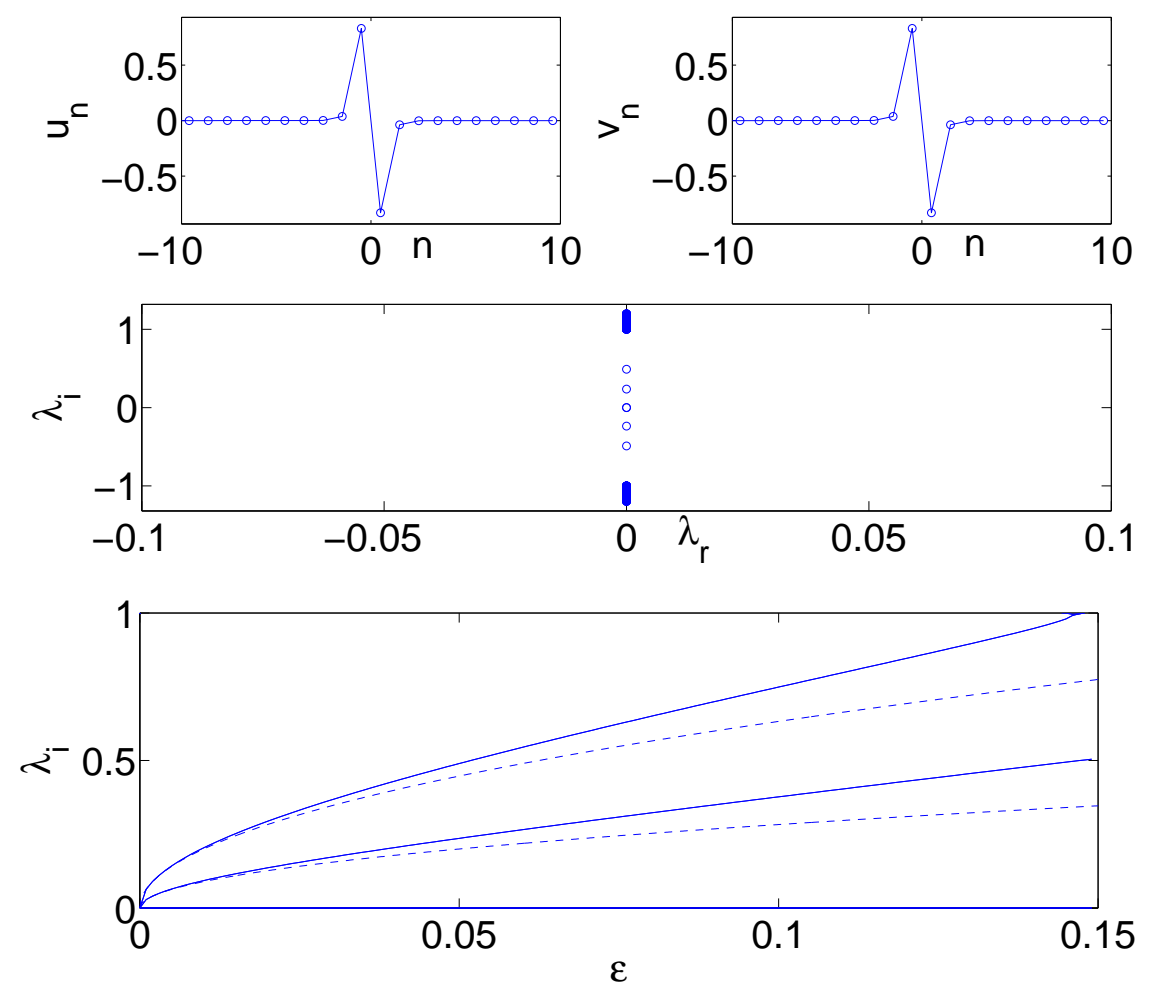

Fig. 4. The top panel shows the mode,+-+- and the spectral plane for $\epsilon=0.05$. The bottom subplot shows the imaginary part of the eigenvalues, for which the theoretical analysis yields $\lambda^{2}=-4 \epsilon,-\frac{4}{5} \epsilon$.

generated as can be seen in the case example of the continuation shown in Fig 2.

- +-,+-: The mode has two purely imaginary eigenvalues

$$
\begin{aligned}
& \lambda_{1}=\sqrt{-\frac{4\left(g_{11}-g_{12}\right)\left(g_{12}-g_{22}\right)}{g_{12}^{2}-g_{11} g_{22}} \epsilon} \\
& \lambda_{2}=2 \sqrt{-\epsilon} .
\end{aligned}
$$

This is once again mandated by the existence constraints considered previously. As a result, similarly to the one-component analog of this out-ofphase mode, in this two-component installment too, we find that this mode is linearly stable in the vicinity of the anti-continuum limit. This mode is only destabilized for sufficiently large values of $\epsilon \approx 0.147$, when one of its imaginary eigenvalues collides with the continuous spectrum and leads to a complex eigenvalue quartet. The relevant detailed results for the continuation of this branch are shown in Fig. 4.

-,+++0 and +-,+0: When $U_{n}$ is an excited site but $V_{n}$ is non-excited for some $n \in S$, then we have the solutions of Eq. (7) or Eq. (8).

This, in turn, leads to $\mathcal{L}^{(0)} \neq 0$, which induces a nonzero eigenvalue 

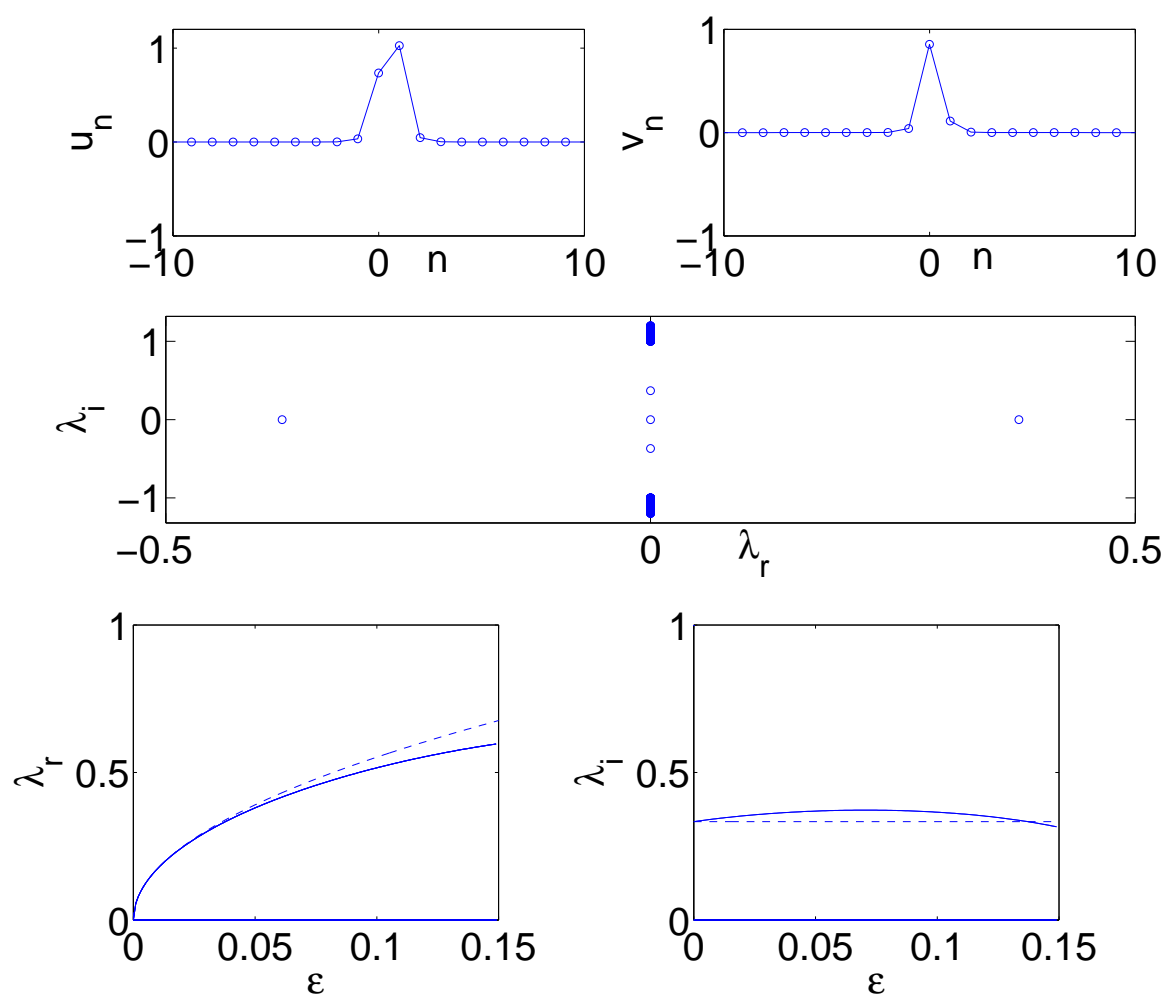

Fig. 5. The top panel shows the mode,+++0 and the spectral plane for $\epsilon=0.05$. The bottom subplot shows the real and imaginary parts of the eigenvalues given (theoretically) by $\lambda=\frac{1}{3} i, 1.74543 \sqrt{\epsilon}$.

$\lambda=\left|\frac{g_{12}}{g_{11}}-1\right| i$ or $\left|\frac{g_{12}}{g_{22}}-1\right| i$, respectively, in the eigenvalue problem (15) of order $\epsilon^{0}$. This already provides the dominant behavior of the corresponding eigenvalues as can be observed in the eigenvalue plots of Figs. 5 and 6 . In addition to this purely imaginary eigenvalue, there is a small eigenvalue bifurcating from the origin in both of these configurations, which is well approximated (for $g_{11}=g_{22}=1$ and $g_{12}=2 / 3$ in the figures), by $\lambda^{2}=$ $\pm 1.74543 \epsilon$. However, this higher order eigenvalue plays a critical role in the stability of the configuration, as in the case of the,+++0 configuration this eigenvalue exists as real (i.e., with the + sign in the above expression), while in the latter case of,+-+0 , it is imaginary. Thus, the former is unstable and the latter is stable, at least for small values of $\epsilon$; for larger $\epsilon$, as can be seen in Fig. 6, a collision arises between the $\mathrm{O}\left(\epsilon^{0}\right)$ near-constant eigenvalue and the growing $\mathrm{O}(\sqrt{\epsilon})$ eigenvalue (between $\epsilon=0.025$ and $\epsilon=0.041$ ) which gives rise to a complex eigenvalue quartet and hence an oscillatory instability.

When $N=3$, analytical expressions for the eigenvalues $\lambda$ are too cumbersome to obtain/write explicitly, however the relevant eigenvalues can be computed (as a function of $\epsilon$ ) by solving a low dimensional eigenvalue problem for each particular set of $g_{i j}$. Fig 7-13 summarize the results for the three modes under 

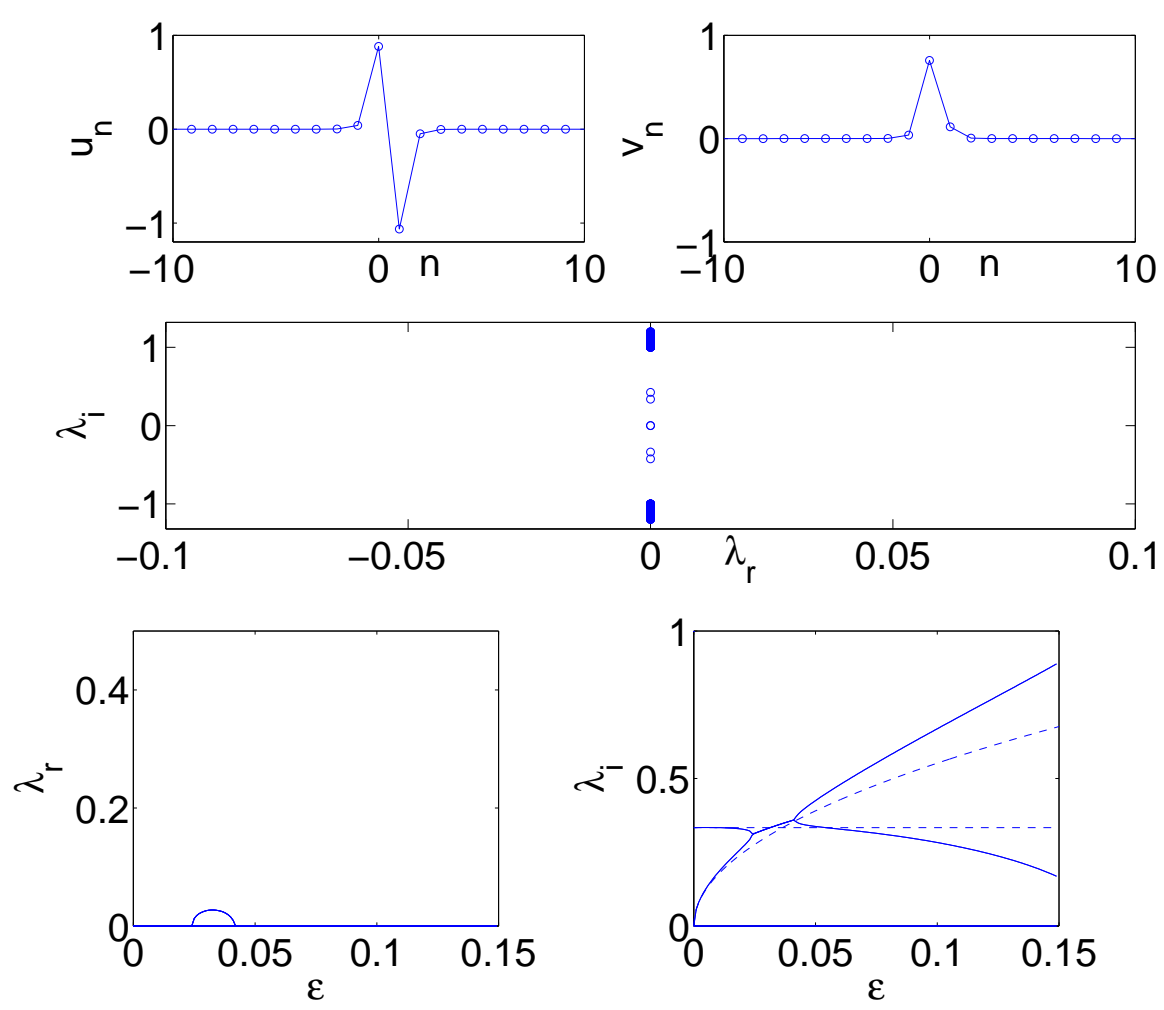

Fig. 6. The top panel shows the mode,+-+0 and the spectral plane for $\epsilon=0.05$. The bottom subplot shows the two pairs of purely imaginary eigenvalues theoretically predicted as $\lambda=1.74543 \sqrt{\epsilon} i, \frac{1}{3} i$. They collide from $\epsilon=0.025$ to $\epsilon=0.041$, i.e., the oscillatory instability is only observed for intermediate values of $\epsilon$ and disappears for larger values of $\epsilon$.

the parameters $g_{11}=g_{22}=1$ and $g_{12}=2 / 3$.

The case of the,++++++ configuration is shown in Fig. 7, where we can again verify the relevant expectations: for each excited site, we expect one pair of emerging eigenvalues, hence 6 pairs should be associated with the 3 excited sites in each component [notice that in the case of 2 excited sites, this amounted to 4 such pairs]. Out of these, 2 are always set at the origin due to the phase invariance of each of the involved components, hence 4 pairs remain as non-vanishing [2 in the 2-excited site case]. The in-phase structure of the configuration renders all of them real in this case in good agreement with the predicted theoretical eigenvalues (of $\lambda^{2}=6 \epsilon, 2 \epsilon, \frac{6}{5} \epsilon, \frac{2}{5} \epsilon$ given by the dashed lines in the graph).

The other two configurations which are identical across components are the ,++-++- of Fig. 8 and the +-+,+-+ of Fig. 9. In direct analogy to their onecomponent counterparts examined in [29], we find that the former of these two configurations bears 2 real pairs of eigenvalues and 2 imaginary ones, while the latter is indeed the only 3-site configuration that enjoys linear stability in the vicinity of the anti-continuum limit, due to the out-of-phase nature of 

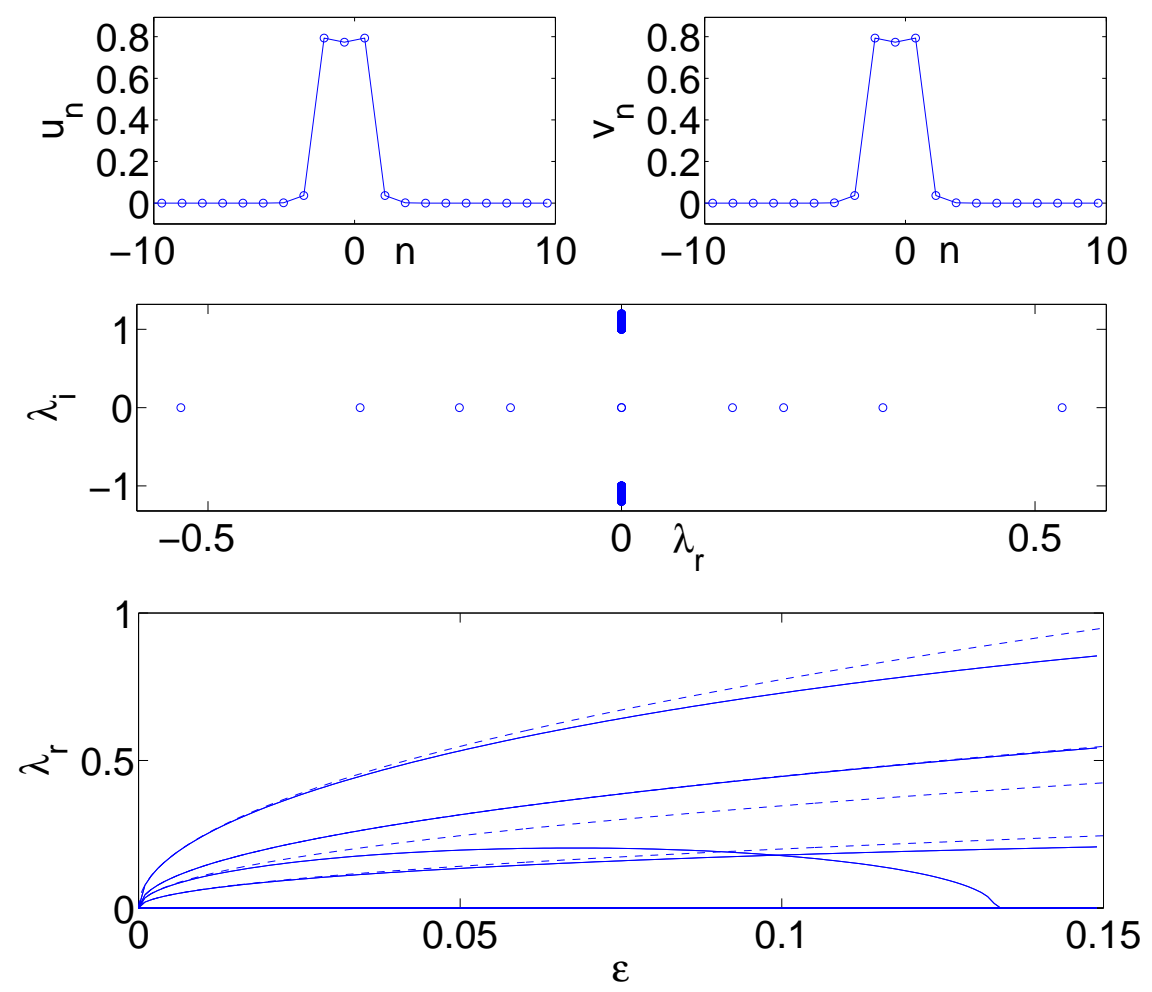

Fig. 7. The mode,++++++ (top panel) with four pairs of real eigenvalues (see the spectral plane of the middle panel) is shown in the figure, captured when $\epsilon=0.05$. The analytical prediction for these pairs $\lambda^{2}=6 \epsilon, 2 \epsilon, \frac{6}{5} \epsilon, \frac{2}{5} \epsilon$ is shown by dashed line in the bottom panel and compared to the numerical results (solid lines).

the neighboring sites therein. In the case of the,++-++- configuration, the eigenvalues are analytically found to be: $\lambda^{2}= \pm 2 \sqrt{3} \epsilon, \pm \frac{2 \sqrt{3}}{5} \epsilon$, while in the case of the,+-++-+ , the $\lambda^{2}$ 's assume the opposite value than they do for the ,++++++ case (yielding 4 imaginary pairs).

Finally, we consider configurations which are distinct in their 3-site form between the two components. These are shown in Figs. 10-13. It should be noted that some of these configurations cease to exist within the interval of $\epsilon$ (up to 0.15$)$ probed herein. In particular, for instance, the mode,+++++- of Fig. 10 collides and disappears with the branch $+0+,++-$, as can be seen in the figure; nevertheless in its interval of existence, we can obtain good agreement with the theoretical prediction for the corresponding eigenvalues $\lambda^{2}=\frac{2}{5}(6 \pm \sqrt{21}) \epsilon, \pm 2 \sqrt{\frac{3}{5}} \epsilon$. In turn, for the mode,++++-+ , we find that it collides with $+0+,+-+$ around $\epsilon=0.06$; the details of the corresponding eigenvalues are shown in Fig. 11. Lastly, the mode,++-+-+ collides with $0+-$ ,+-+ , as shown in Fig. 12, and the mode,++-+- collides and disappears with $0+-,+-0$, as detailed in Fig. 13. 

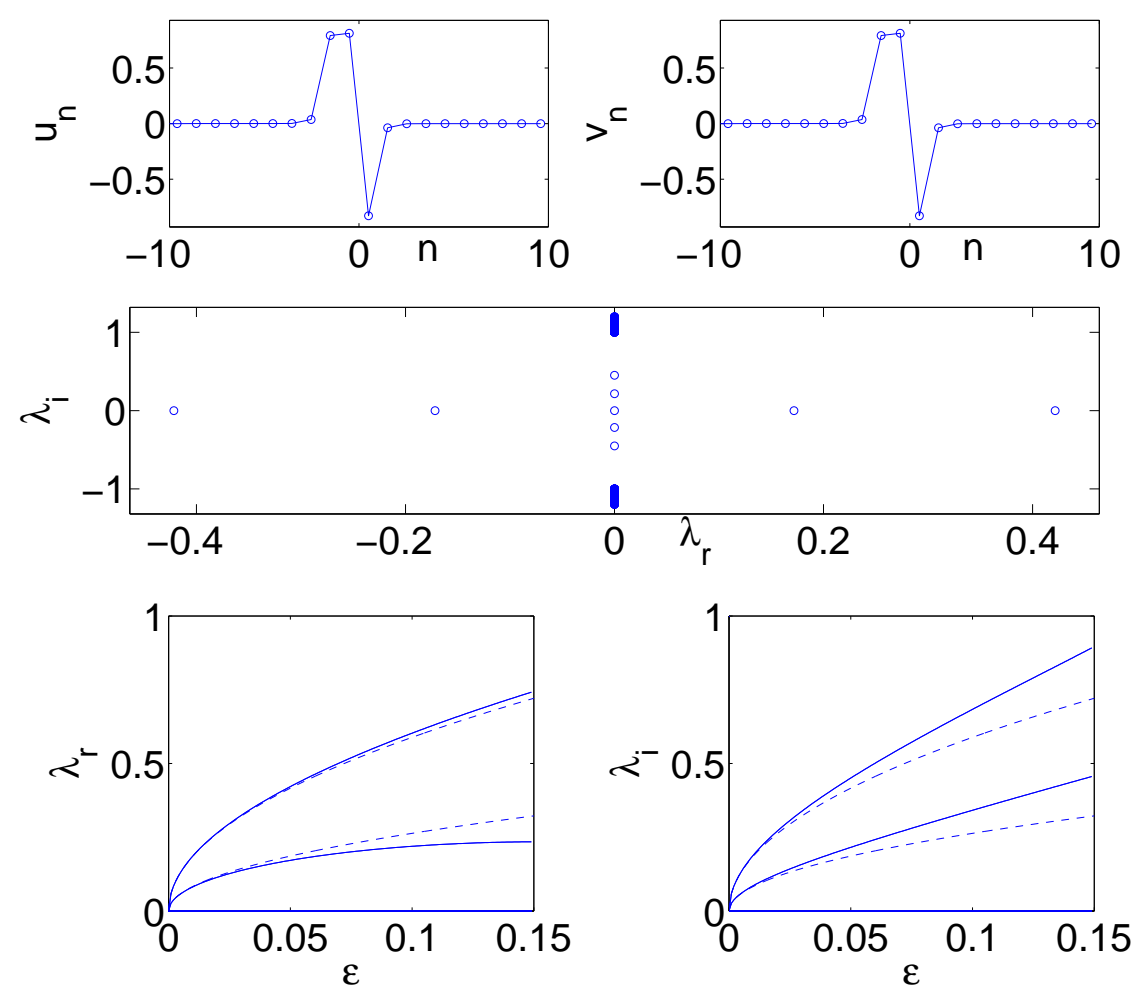

Fig. 8. Similarly to Fig 7 but for the mode,++-++- with two pairs of real and two pairs of purely imaginary eigenvalues given by $\lambda^{2}= \pm 2 \sqrt{3} \epsilon, \pm \frac{2 \sqrt{3}}{5} \epsilon$. The top three panels are captured when $\epsilon=0.05$.

\section{Conclusions \& future challenges}

In the present work, we considered two-component settings and provided a general framework for the existence and stability of localized mode solutions to nonlinear dynamical lattices of the DNLS type. We illustrated that 0 and $\pi$ relative phase configurations arise in this context, in analogy to the case of the one-component DNLS model. Although the stability presented some interesting deviations from the corresponding one-component counterpart (e.g. in the case of the,+++- configuration), most of the cases presented significant analogies in the nature of the stability conclusions (with suitable modifications of the eigenvalue counts) to the one-component setting. In particular, the existence of in-phase near-neighbor excitations in the same component appeared to generically lead to the existence of real eigenvalue pairs in the linearization. On the other hand, the only configurations that seemed to generically be stable were those where all adjacent sites were out of phase with respect to each other. In the case of the,+++- configuration, presented an interesting possibility. In particular, depending on the relation between $g_{11}, g_{22}$ and $g_{12}$, the waveform may feature either exponential (as ++ ) or oscillatory (which +would possess but far from the AC limit) instabilities as soon as the coupling 

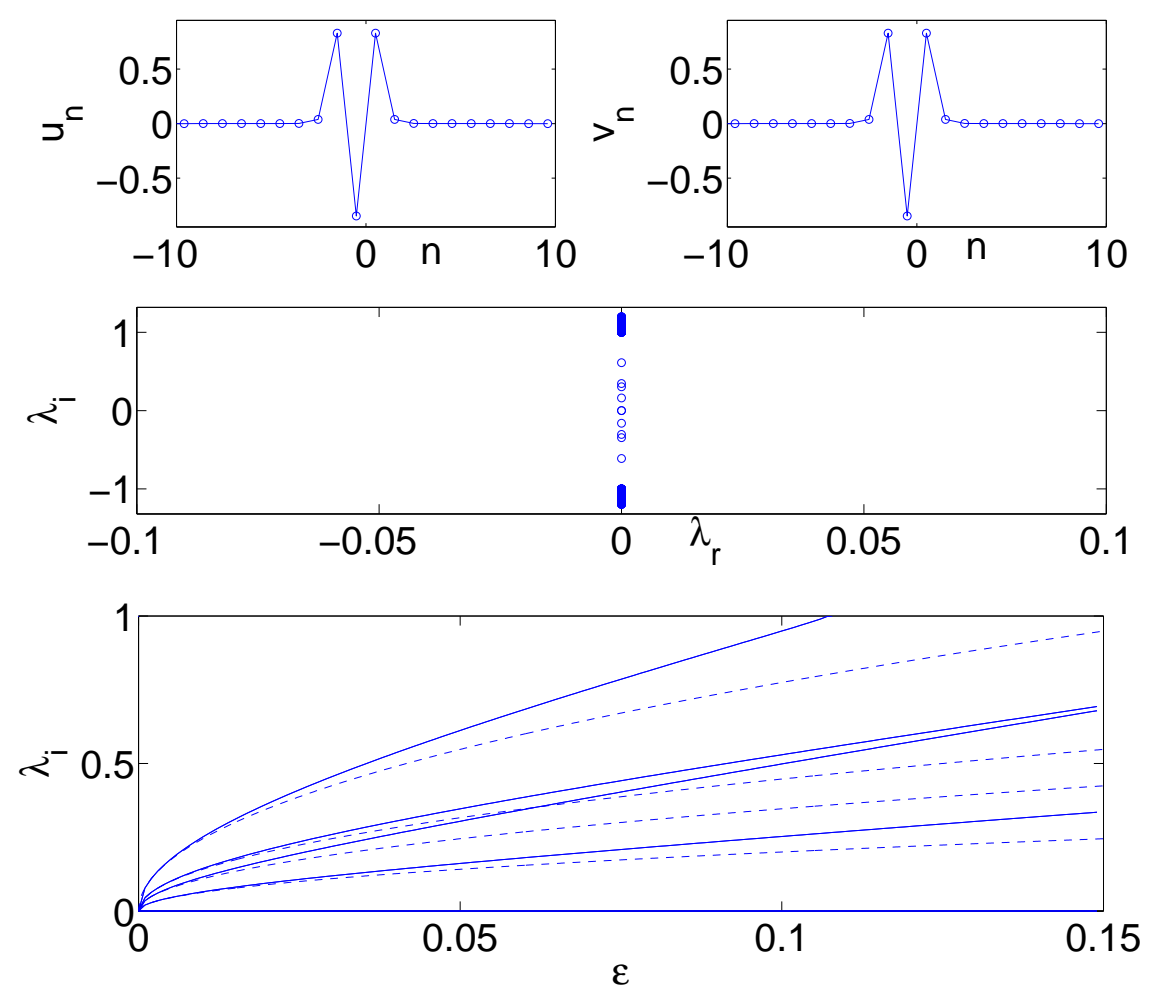

Fig. 9. Similarly to Fig 7 but for the mode,+-++-+ with four pairs of purely imaginary eigenvalues theoretically given by $\lambda^{2}=-6 \epsilon,-2 \epsilon,-\frac{6}{5} \epsilon,-\frac{2}{5} \epsilon$. The top three panels are captured when $\epsilon=0.05$.

strength becomes nonzero. This is a case where the inter-component coupling affects the specific type of instability observed (and can thus modify it in comparison to the single component case). On the other hand, more broadly, a global consideration of the states analyzed herein suggests that we can think of the stability (1) or instability (0) of each component as a logical input. In that framework, the stability of the two component system operates in the form of an "AND" gate, and indeed necessitates the stability of the relevant constituents.

It is natural to envision numerous generalizations of the present considerations. While already some special case examples of discrete vortex configurations have been examined in [19], a general theory of both discrete solitons and of vortices is still absent in the two-component setting. Far less work has been done in the three-dimensional case, where identifying suitable stable configurations with nontrivial phase profiles is a fairly challenging task even in the one-component case [21]. While these considerations are still lacking for the more standard DNLS model, this is certainly all the more so the case for the setting with four-wave mixing terms being present, as appears to be experimentally relevant per the work of [22]. Finally, another possibility than the incoherent (phase-independent) coupling presented herein or the 

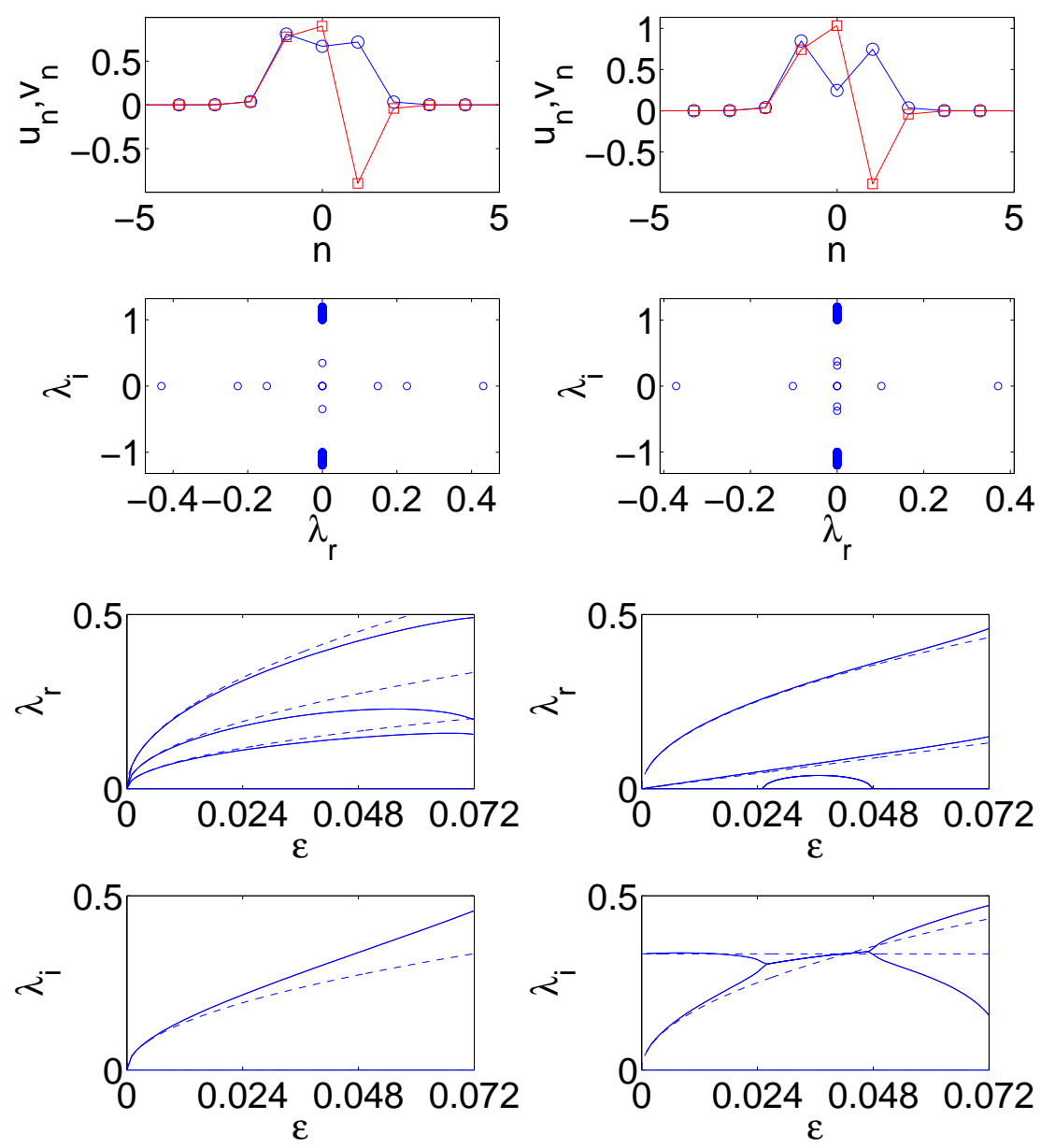

Fig. 10. The left column shows the mode,+++++- , which terminates at $\epsilon=0.072$ by colliding with the mode $+0+,++-$ shown in the right column. The top two rows show the profile and the linear stability of each mode when $\epsilon=0.05$. The bottom two rows show the eigenvalues of each mode. The mode,+++++- has three pairs of real and one pair of purely imaginary eigenvalues given by $\lambda^{2}=\frac{2}{5}(6 \pm \sqrt{21}) \epsilon, \pm 2 \sqrt{\frac{3}{5}} \epsilon$, while the mode $+0+,++-$ has two pairs of real and two pair of purely imaginary eigenvalues given by $\lambda=\frac{1}{3} i, 1.61812 \sqrt{\epsilon} i, 1.61812 \sqrt{\epsilon}, 1.82599 \epsilon$. The first two pairs of eigenvalues collide and form a complex eigenvalue quartet from $\epsilon=0.025$ to 0.048 .

four-wave mixing nonlinear coupling mentioned above is the case where the two components sustain linear coupling between them. In the latter case, even for single-site excitations interesting phenomenologies have been illustrated e.g. in [15], such as symmetry breaking and pitchfork type bifurcations. It would thus be especially relevant in that regard to examine multi-site modes and how their stability properties will (potentially dramatically due to the phase-coherent coupling) change in comparison to the findings herein. Some of these avenues are presently being considered and will be reported in future publications. 

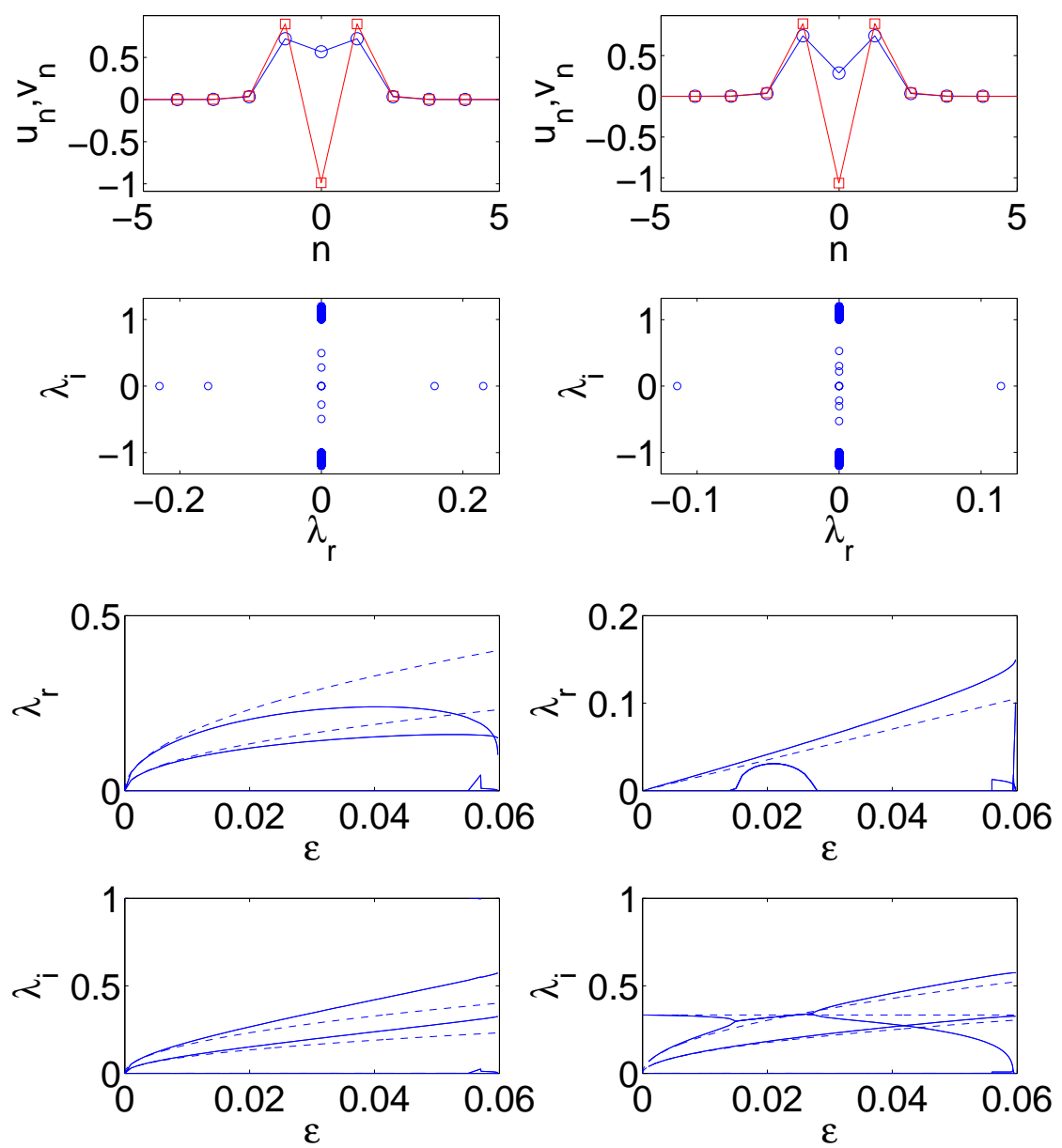

Fig. 11. Similar as Fig. 10 but here the left column shows the mode,++++-+ , which has eigenvalues $\lambda^{2}= \pm \frac{2}{\sqrt{5}} \epsilon, \pm \frac{6}{\sqrt{5}} \epsilon$ and the right column contains the mode $+0+,+-+$, which has eigenvalues $\lambda=\frac{1}{3} i, 1.24467 \sqrt{\epsilon} i, 2.14026 \sqrt{\epsilon} i, 1.75819 \epsilon$ (according to the theoretical analysis). The first two pairs of eigenvalues collide and form an eigenvalue quartet from $\epsilon=0.014$ to 0.028 . The top four panels are captured when $\epsilon=0.05$.

Acknowledgments. VR research has been co-financed by the European Union (European Social Fund ESF) and Greek national funds through the Operational Program "Education and Lifelong Learning" of the National Strategic Reference Framework (NSRF) - Research Funding Program: THALES. Investing in knowledge society through the European Social Fund. PGK gratefully acknowledges support from the National Science Foundation through grants DMS-0806762 and CMMI-1000337, as well as from the Alexander von Humboldt Foundation, the Alexander S. Onassis Public Benefit Foundation and the Binational Science Foundation. 

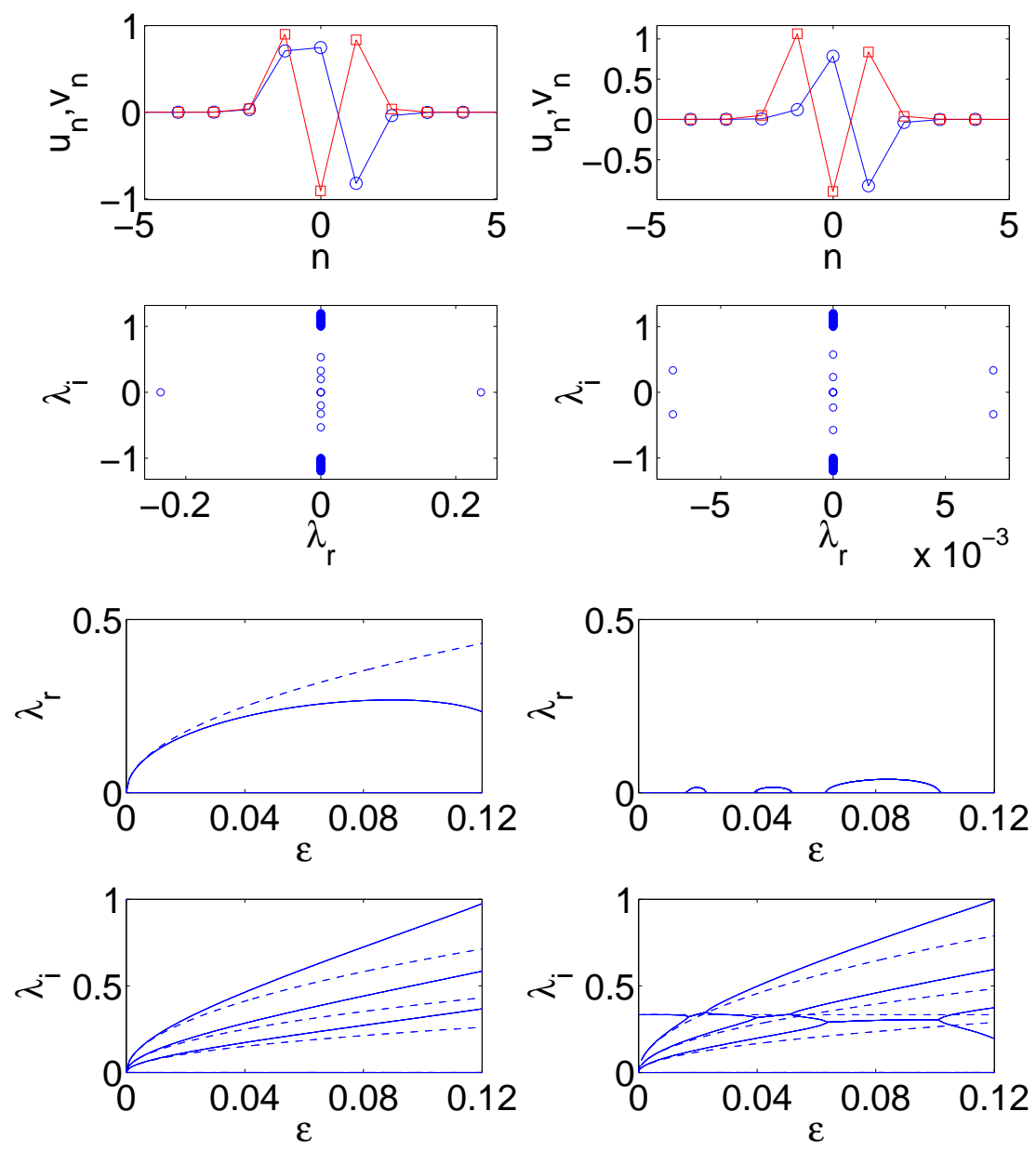

Fig. 12. Similar as Fig. 10 but here the left column has the mode,++-+-+ , which has eigenvalues $\lambda^{2}=-\frac{2}{5}(6 \pm \sqrt{21}) \epsilon, \pm 2 \sqrt{\frac{3}{5}} \epsilon$ while the right column contains the mode $0+-,+-+$, which has four pairs of purely imaginary eigenvalues given by $\lambda=\frac{1}{3} i, 0.834064 \sqrt{\epsilon} i, 1.39576 \sqrt{\epsilon} i, 2.27766 \sqrt{\epsilon} i$. The eigenvalues of order $\epsilon^{0}$ collide with the others from $\epsilon=0.016$ to 0.023 , from 0.039 to 0.052 , and from 0.063 to 0.102 , respectively, giving rise to the corresponding oscillatory instabilities. The top four panels are captured when $\epsilon=0.05$.

\section{References}

[1] G. Alfimov, V. Brazhnyi, V. Konotop, On classification of intrinsic localized modes for the discrete nonlinear schrodinger equation, Physica D 194 (2004) 127.

[2] S. Aubry, Breathers in nonlinear lattices: Existence, linear stability and quantization, Physica D 103 (1997) 201.

[3] V. Brazhnyi, V. Konotop, Theory of nonlinear matter waves in optical lattices, Mod. Phys. Lett. B 18 (2004) 627. 

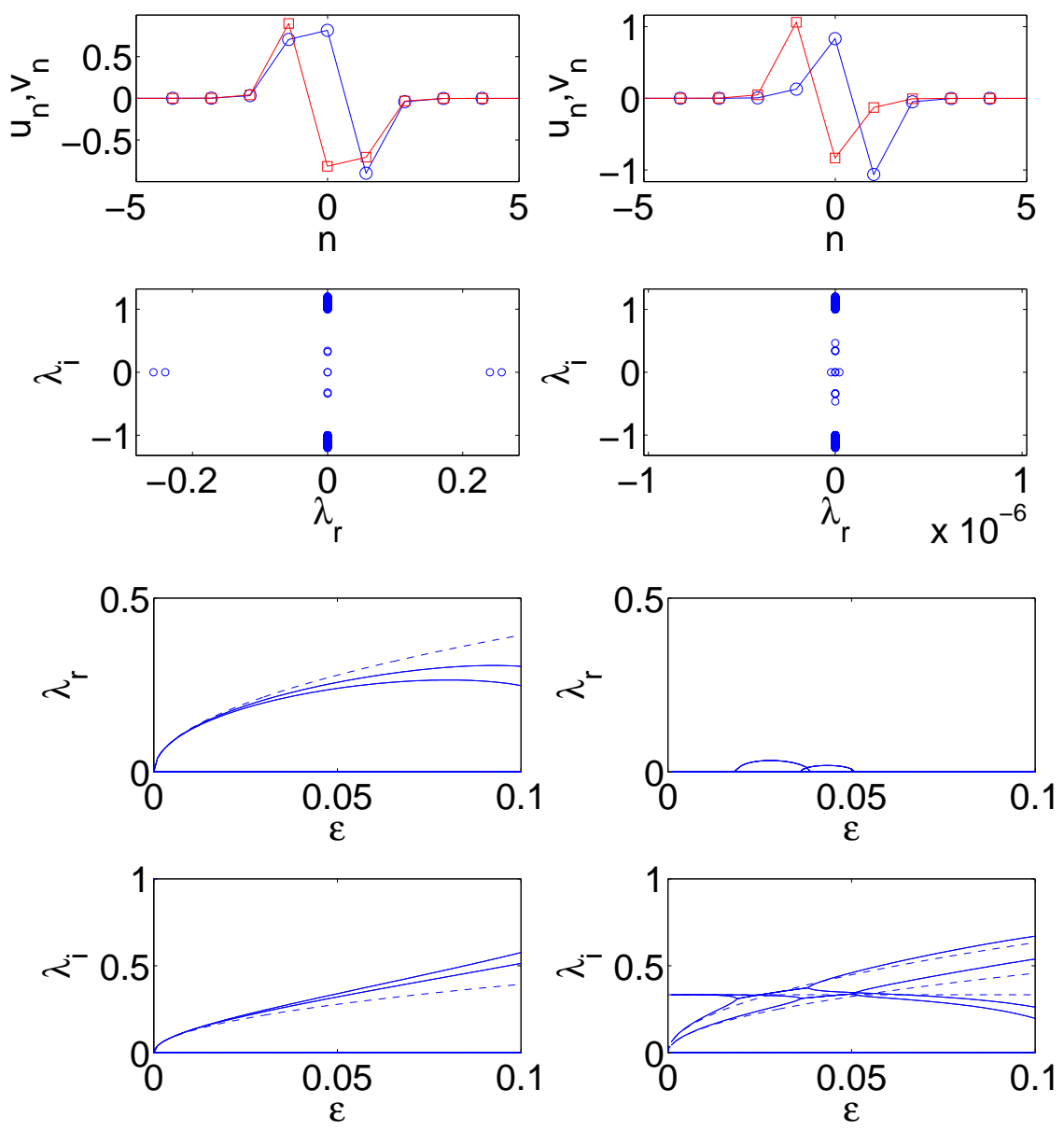

Fig. 13. Similar as Fig. 10 but the left column shows the mode,++-+- , which has four pairs of eigenvalues $\lambda^{2}= \pm 2 \sqrt{\frac{3}{5} \epsilon}, \pm 2 \sqrt{\frac{3}{5} \epsilon}$, while the right column contains the mode $0+-,+-0$, which has four pairs of purely imaginary eigenvalues given by $\lambda=\frac{1}{3} i, \frac{1}{3} i, 1.44578 \sqrt{\epsilon} i, 2.00338 \sqrt{\epsilon} i$. The eigenvalues of order $\epsilon^{0}$ collide with the others from $\epsilon=0.018$ to 0.039 , from 0.036 to 0.051 , respectively, creating once again an oscillatory instability. The top four panels are captured when $\epsilon=0.05$.

[4] Z. Chen, J. Yang, A. Bezryadina, I. Makasyuk, Observation of two-dimensional lattice vector solitons, Opt. Lett. 29 (2004) 1656.

[5] D. N. Christodoulides, F. Lederer, Y. Silberberg, Discretizing light behaviour in linear and nonlinear waveguide lattices, Nature 424 (2003) 817-823.

[6] J. Cuevas, Q. Hoq, H. Susanto, P. Kevrekidis, Interlaced solitons and vortices in coupled dnls lattices, Physica D 238 (2009) 2216.

[7] R. Dong, C. Rüter, D. Kip, J. Cuevas, P. Kevrekidis, D. Song, J. Xu, Dark-bright gap solitons in coupled-mode one-dimensional saturable waveguide arrays, Phys. Rev. A 83 (2011) 063816.

[8] N. K. Efremidis, S. Sears, D. N. Christodoulides, J. W. Fleischer, M. Segev, Discrete solitons in photorefractive optically induced photonic lattices, Phys. 
Rev. E 66 (2002) 046602.

[9] H. Eisenberg, Y. Silberberg, R. Morandotti, A. R. Boyd, J. S. Aitchison, Discrete spatial optical solitons in waveguide arrays, Phys. Rev. Lett. 81 (1998) 3383 .

[10] S. Flach, A. Gorbach, Discrete breathers - advances in theory and applications, Phys. Rep. 467 (2008) 1.

[11] S. Flach, C. R. Willis, Discrete breathers, Phys. Rep. 295 (1998) 181.

[12] J. Fleischer, G. Bartal, O. Cohen, T. Schwartz, O. Manela, B. Freedman, M. Segev, H. Buljan, N. Efremidis, Spatial photonics in nonlinear waveguide arrays, Optics Express 13 (2005) 1780.

[13] J. W. Fleischer, T. Carmon, M. Segev, N. K. Efremidis, D. N. Christodoulides, Observation of discrete solitons in optically induced real time waveguide arrays, Phys. Rev. Lett. 90 (2003) 023902.

[14] J. W. Fleischer, M. Segev, N. K. Efremidis, D. N. Christodoulides, Observation of two-dimensional discrete solitons in optically-induced nonlinear photonic lattices, Nature 422 (2003) 147.

[15] G. Herring, P. G. Kevrekidis, B. A. Malomed, R. Carretero-González, D. J. Frantzeskakis, Symmetry breaking in linearly coupled dynamical lattices, Phys. Rev. E 76 (2007) 066606.

[16] J. Hudock, P. Kevrekidis, B. Malomed, D. Christodoulides, Discrete vector solitons in two-dimensional nonlinear waveguide arrays: Solutions, stability, and dynamics, Phys. Rev. E 67 (2003) 056618.

[17] P. Kevrekidis, The Discrete Nonlinear Schrodinger Equation: Mathematical Analysis, Numerical Computations and Physical Perspectives, Springer-Verlag, Heidelberg, 2009.

[18] P. Kevrekidis, D. Frantzeskakis, R. Carretero-González (eds.), Emergent Nonlinear Phenomena in Bose-Einstein Condensates: Theory and Experiment, Springer-Verlag, Heidelberg, 2008.

[19] P. Kevrekidis, D. Pelinovsky, Discrete vector on-site vortices, Proc. Roy. Soc. London A 462 (2006) 2671.

[20] F. Lederer, G. Stegeman, D. Christodoulides, G. Assanto, M. Segev, Y. Silberberg, Discrete solitons in optics, Phys. Rep. 463 (2008) 1.

[21] M. Lukas, D. Pelinovsky, P. Kevrekidis, Lyapunov-schmidt reduction algorithm for three-dimensional discrete vortices, Physica D 237 (2008) 339.

[22] J. Meier, J. Hudock, D. Christodoulides, G. Stegeman, Y. Silberberg, R. Morandotti, J. Aitchison, Discrete vector solitons in kerr nonlinear waveguide arrays, Phys. Rev. Lett. 91 (2003) 143907. 
[23] K. Mertes, J. Merrill, R. Carretero-González, D. Frantzeskakis, P. Kevrekidis, D. Hall, Nonequilibrium dynamics and superfluid ring excitations in binary bose-einstein condensates, Phys. Rev. Lett. 99 (2007) 190402.

[24] G. Modugno, G. Ferrari, G. Roati, R. Brecha, A. Simoni, M. Inguscio, Boseeinstein condensation of potassium atoms by sympathetic cooling, Science 294 (2001) 1320.

[25] O. Morsch, E. Arimondo, Dynamics and Thermodynamics of Systems with Long-Range Interactions, Springer, Berlin, 2002.

[26] O. Morsch, M. Oberthaler, Dynamics of bose-einstein condensates in optical lattices, Rev. Mod. Phys. 78 (2006) 179.

[27] M. Mudrich, S. Kraft, K. Singer, R. Grimm, A. Mosk, M. Weidemüller, Sympathetic cooling with two atomic species in an optical trap, Phys. Rev. Lett. 88 (2002) 253001.

[28] C. J. Myatt, E. Burt, R. Ghrist, E. Cornell, C. Wieman, Production of two overlapping bose-einstein condensates by sympathetic cooling, Phys. Rev. Lett. 78 (1997) 586.

[29] D. Pelinovsky, P. Kevrekidis, D. Frantzeskakis, Stability of discrete solitons in nonlinear schrödinger lattices, Physica D 212 (2005) 1.

[30] D. Stamper-Kurn, M. Andrews, A. Chikkatur, H.-J. M. S. Inouye, J. Stenger, W. Ketterle, Optical confinement of a bose-einstein condensate, Phys. Rev. Lett. 80 (1998) 2027. 